\title{
HMGB1 and sRAGE: What are Their Links to Acute Myeloid Leukemia?
}

Zaridatul Aini Ibrahim ${ }^{1}$, Kim Jun Cheng1, Noor Atiqah Fakaruzi1,2, Ariwibawa Kasmani Mohd Hashim ${ }^{1,2}$, Shamsul Mohd Zain ${ }^{1}$, Ezalia Esa², Yuslina Mat Yusoff ${ }^{2}$, Nor Rizan Kamaluddin², Zubaidah Zakaria², Elsa Haniffah Mejia Mohamed ${ }^{1}$

${ }^{1}$ Department of Pharmacology, Faculty of Medicine, University Malaya, 50603 Kuala Lumpur; ${ }^{2}$ Haematology Unit, Cancer Research Centre, Institute for Medical Research, Jalan Pahang, 50588 Kuala Lumpur, Malaysia

\begin{abstract}
Introduction: Acute myeloid leukemia (AML) is a disparate hematopoietic malignancy, with many subtypes and variable responsiveness to therapy. Identification of a targetable biomarker can be used to improve survival outcome of this disease. High mobility group box 1 (HMGB1), contributes to the progression of AML by inducing various cytokines production and angiogenic vascular endothelial growth factor (VEGF). Receptor for advanced glycation end products (RAGE) exists as both a membrane and soluble receptor (sRAGE). HMGB1 is a ligand for RAGE, with sRAGE serving as a natural antagonist of RAGE signaling. Accumulating evidence suggests that HMGB1/sRAGE axis is critically involved in many cancers.
\end{abstract}

Objective: The aim of this study was to investigate whether there is a difference in the plasma levels of HMGB1/sRAGE between AML patients with good and poor prognosis and those who go into relapse.

Methods: A total of 97 plasma samples from peripheral blood were analysed for the study (47 AML, 50 healthy subjects). HMGB1 and sRAGE levels were measured using ELISA and analysed using GraphPad Prism 5.0.

Results: HMGB1 levels were found to be significantly higher in relapsed AML cases as compared to newly diagnosed cases $(49.81 \pm 25.25(\mathrm{~N}=11)$ vs $9.461 \pm 4.770(\mathrm{~N}=36), \mathrm{pg} / \mathrm{ml}, \mathrm{p}$ =0.0161). HMGB1 was also significantly higher among new AML cases classified as having poor prognosis as compared to good prognosis $(26.11 \pm 13.31(\mathrm{~N}=12)$ vs $1.135 \pm 0.7147$ $(\mathrm{N}=24), \mathrm{pg} / \mathrm{ml}, \mathrm{p}=0.0114)$. Although sRAGE levels were also high where HMBG1 levels were high, the differences were not significant.

Conclusion: HMGB1 may be used as a prognostic biomarker, as it appears to promote AML progression with higher levels seen in more malignant forms. Unravelling the mechanisms of HMGB1 in the pathogenesis of AML could provide means to regulate its expression and activities and serve as an effective treatment for more aggressive subtypes.

Keywords: AML; HMGB1; sRAGE 